\title{
Study of mental well being among public sector employees in India using a mobile based psychiatric assessment tool
}

S. Krishna ${ }^{1}$, P. Upadhyay ${ }^{2}$, V. Sharma ${ }^{3}$.

${ }^{1}$ Independant Practitioner- Consultant Psychiatrist, Psychiatry, Bangalore, India.

${ }^{2}$ Atal Bihari Vajpayee Institute of Good Governance and Policy Analysis, State Health

Resource Centre- Social Sector Department, Bhopal, India.

${ }^{3}$ University of Chester, Psychiatry, Chester, United Kingdom.

\section{Objectives:}

The aim of the study was to understand the emotional well being of public sector employees in a semi urban set up in India.

\section{Background:}

According to the National Mental Health survey (2016) in India, the lifetime prevalence of mental disorders was $13.7 \%$. The WHO report that depression is one of the leading causes of disability worldwide. This suggests that the economic burden of mental disorders in India is high.

\section{Materials and Methods:}

This was a cross-sectional study conducted in October 2018 in Bhopal, India. The participants were from different departments in public sector. The survey was conducted by doctors in alternative medicine using a mobile based psychiatric assessment tool called Global Mental Health Assessment Tool - Primary Care Version (GMHAT/PC). A work satisfaction questionnaire was used to understand working conditions and work hours. Informed consent was obtained from all participants.

\section{Results and Conclusions:}

Results: Out of a total of 1391 participants, $56 \%$ were female. $41 \%$ of the study participants had a mental disorder - most common was anxiety (17\%) followed by depression (12 percent). $26 \%$ of the participants worked longer than 12 hours. The presence of mental disorders was 57\% in the group that worked longer than 12 hours and this was significantly higher than those working between 8 to 11 hours.

\section{Conclusions:}

Our study showed a higher prevalence of depression and anxiety. There was a correlation between longer working hours and mental disorders. This highlights the need to integrate regular assessments of well being including mental health within large organisations. 